\title{
Malária em paciente transplantado renal: Uma nova ótica para um problema antigo - Relato de caso
}

\author{
Malaria in a renal transplant patient: A new approach to an old problem - case report
}

Diego de Oliveira Santos $†$, Géssika Marcelo Gomes ${ }^{\ddagger}$ Carlos Eduardo Cardoso ${ }^{\circledR}$

Como citar esse artigo. Santos DO;

Gomes GM; Cardoso CE. Malária em paciente transplantado renal Uma nova ótica para um problema antigo - Relato de caso . Revista de Saúde. 2018 Jan./Jun.; 09 (1): 22-25

\begin{abstract}
Resumo
A febre terçã ou malária é uma doença infecciosa endêmica da América Latina, África e Ásia e afetou em 2015 cerca de 212 milhões de pessoas, sendo que 459 mil pessoas foram a óbito. A malária é uma infecção considerada rara em transplantados renais e sua evolução é pouco descrita na literatura. O objetivo deste trabalho foi relatar esta doença em paciente transplantado renal de longa data e suas peculiaridades. Este paciente transplantado renal contraiu malária em fevereiro de 2017 em um garimpo de ouro na Guiana Francesa e foi tratando com Artesunato. Em março de 2017, após tratamento, retornou com sinais e sintomas e com comprovação de infecção por Plasmodium vivax através de exame laboratorial de gota espessa. Na sequência, o paciente foi tratado com cloroquina ( 3 dias) e primaquina (18 dias) com boa evolução clínica.

Palavras-chave: Malária; Transplantado Renal; Doenças Negligenciadas.
\end{abstract}

\begin{abstract}
The third fever or malaria is an infectious disease endemic in Latin America, Africa and Asia and affected in 2015 about 212 million and 459 thousand people died. The objective of this study was to report malaria in a long-term renal transplant patient and its peculiarities. Renal transplant patient contracted malaria in February 2017 in gold panning in French Guiana, dealing with Artesunato. In March 2017 even after treatment returned with signs and symptoms, with evidence of infection by Plasmodium vivax through laboratory examination of thick gout was treated with good clinical evolution. Malaria infection in renal transplant patients is considered rare and its evolution is poorly understood by the scientific community.

Keywords: Malaria; Kidney Transplanted; Neglected Diseases.
\end{abstract}

impaludismo, paludismo e febre terçã (benigna ou maligna) ou quartã, é uma doença infecciosa febril aguda, causada por protozoários (Plasmodium vivax, Plasmodium falciparum, Plasmodium malariae e Plasmodium ovale) transmitidos pela fêmea infectada do mosquito do gênero Anopheles Meigen. Apresenta uma sintomatologia típica constituída por episódios de calafrios, seguidos de febre alta que duram de três a quatro horas. Esses episódios são acompanhados de profundo mal-estar, náuseas, cefaleias e dores articulares ${ }^{1,2}$. O implaudismo é uma endemia altamente prevalente no mundo afetando cerca de 212 milhões de pessoas, havendo 429 mil mortes pela doença em regiões como África, Ásia e America Latina em 2015 $\mathrm{Na}$ América do Sul, a floresta amazônica é a região com mais casos registrados da doença no continente, afetando países como Suriname, Guiana, Guiana Francesa, Brasil e Venezuela, apesar dos mesmos terem apresentado uma queda de $20 \%$ no número de novos casos em 20154.

No Brasil, mais de $99 \%$ dos casos notificados de malária estão registrados na região amazônica, que engloba os estados do Acre, Amazonas, Amapá, Maranhão, Mato Grosso, Pará, Rondônia, Roraima e Tocantins. Nessa região há condições propícias para a sobrevivência dos vetores e as condições socioeconômicas e ambientais favorecem a transmissão da doença $a^{3,5}$.

Apesar da alta taxa de contaminação em pessoas de diversas idades em vários continentes, a malária ainda é considerada uma infecção rara em pacientes transplantados renais. Esta população específica realiza uso de medicamentos (imunossupressores) que 
as deixam mais suscetíveis a infecções parasitárias. Além desse risco devido à imunossupressão deve-se acrescentar a possibilidade de infecção via aloenxerto caso não haja uma triagem adequada no momento do transplante. A infecção de pacientes transplantados renais pode ser considerada rara por dois possíveis motivos: baixa divulgação em meio científico ou pelo fato dos grandes centros transplantadores não se localizarem em áreas endêmicas de malária. Assim, o objetivo deste trabalho foi relatar um caso de malária em paciente transplantado renal de longa data e suas peculiaridades $6,7,8$.

Um paciente de 47 anos do gênero masculino, católico, pardo, minerador, natural de Mossoró (RN) e residente em Guarulhos (SP), portador de doença renal crônica (DRC) de etiologia indeterminada, hipertensão arterial sistêmica, transplantado renal de doador vivo há 13 anos e 10 meses, com creatinina estável em $1,1 \mathrm{mg} / \mathrm{dL}$. O paciente fazia uso regular de imunossupressão oral (150 mg de ciclosporina, $5 \mathrm{mg}$ de prednisona e $100 \mathrm{mg}$ de azatioprina) e ácido acetil salicílico (100 mg), $50 \mathrm{mg}$ de losartan de $12 \mathrm{em} 12 \mathrm{~h}, 20$ $\mathrm{mg}$ de monocordil às $8 \mathrm{~h} \mathrm{e} \mathrm{16h,} 25 \mathrm{mg}$ de atenolol, 400 $\mathrm{mg}$ de sulfametoxazol, $80 \mathrm{mg}$ de trimetopim e $150 \mathrm{mg}$ de ranitidina de $12 \mathrm{em} 12 \mathrm{~h}$.

Conforme relatou na anamnese, o paciente viajou para a Guiana Francesa em dezembro de 2016 para trabalhar em um garimpo onde permaneceu por três meses. Em fevereiro de 2017 iniciou quadro febril e dores musculares, procurando assistência médica local que o diagnosticou com malária e o tratou com Artefan ${ }^{\circledR}$ por quatro dias.

Retornando ao Brasil em março de 2017, permaneceu sem sinais ou sintomas até o dia 26/03/2017, quando foi admitido no pronto socorro do Hospital do Rim na cidade de São Paulo com febre aferida de $40^{\circ} \mathrm{C}$ associada à cefaleia holocraniana, calafrios e inapetência. Ao exame estava hipotenso (90X60 $\mathrm{mmHg})$, apresentava uma hiperemia conjuntival e a ausculta cardíaca e respiratória não revelou nenhuma alteração. $\mathrm{O}$ abdomedo paciente apresentava-se doloroso à palpação em hipocôndrios direito e esquerdo e região epigástrica. Em função disto, o paciente foi internado e foi realizada a estabilização hemodinâmica, além da aplicação de medicação para os sintomas. Complementarmente, foram solicitados exames de ultrassonografia de abdome total que evidenciou fígado, vias biliares, pâncreas, baço, bexiga e vesícula biliar sem alterações e rim transplantado localizado na fossa ilíaca direita com forma, contornos e dimensões normais e exame de gota espessa, que resultou positivo para malária causada por $P$. vivax $(++)$.

O paciente iniciou o tratamento para malária com cloroquina $(150 \mathrm{mg})$, ingerindo quatro comprimidos por dia durante três dias e posteriormente, em tratamento domiciliar, um comprimido por dia de primaquina $(15 \mathrm{mg})$ durante 18 dias. Tal abordagem resultou em evolução com melhora da creatinina (que retornou ao valor basal) e resolução dos sintomas. Segue em acompanhamento trimestral no ambulatório de póstransplante no Hospital do Rim em São Paulo.

A malária é uma enfermidade infecciosa cujo agente etiológico é um parasita do gênero Plasmodium. As variantes do Plasmodium associada àpatologia em humanos encontram-se as espécies $P$. falciparum, $P$. vivax, o $P$. malariae e $P$. ovale, sendo a última variação restrita a certos territórios na África, não havendo relatos de casos de contaminação em outras regiões ${ }^{1,6}$. A contaminação se dá pela picada do mosquito vetorno humano, depositando na corrente sanguínea os esporozoítos que buscam invadir as células hepáticas. Os esporozoítos dentro das células hepáticasse modificam para merozoítos, estes últimos invadem as hemácias e começa o processo de replicação do protozoário.Após há a liberação dos merozoítos para a corrente sanguínea onde ocorre nova invasão das hemácias (nesta fase surgemos sinais e sintomas da malária) ${ }^{1,6}$.Tais eventos ocorrem em torno de uma semana tanto pela infecção do $P$. falciparum quanto do $P$. vivax e duas semanas pelo $P$. malariae $^{1,3,6} . \mathrm{O}$ tempo de incubação pode variar de 7 a 14 dias ou se estender a meses, variando de acordo com acondição imunológica de cada paciente ${ }^{1,2}$. Os sinais e sintomas causados pela infecção são,classicamente, calafrios, febre e sudorese que têm duração variável de 6 a 12 horas, podendo cursar com temperatura igual ou superior a $40^{\circ} \mathrm{C}$, acompanhados por cefaleia, mialgia, náuseas e vômitos ${ }^{1,3,4}$. Após os primeiros dias, a febre se torna do tipo intermitente e, se houver infecção pelo $P$. vivax, apresentará quadros febris a cada 48 horas(febre terçã benigna). Se contaminado pelo $P$. falciparum, a febre terá espaços de 36 a 48 horas (terçã maligna) entre um episódio e outro se a infecção for pelo $P$. malariae, que se caracteriza pela presença de febre a cada 72 horas (febre quartã) $)^{17}$. O diagnóstico da doença se dá pela visualização direta do parasita na corrente sanguínea do paciente através do exame da gota espessa.O quadro pode ser agravado pelas comorbidades e estado imunológico do paciente infectado. Idosos, crianças e gestantes tem maior risco de evolução desfavorável ${ }^{1,2}$. A malária grave caracteriza-se por alteração da consciência, dispneia ou hiperventilação, convulsões, hipotensão arterial ou choque, edema pulmonar na radiografia de tórax, hemorragias, icterícia, hemoglobinúria, hiperpirexia $\left(>41^{\circ} \mathrm{C}\right)$ e oligúria.

A malária está no quadro de doenças negligencia das da Organização Mundial da Saúde $(\mathrm{OMS})^{4}$, isto é, patologias que possuem baixo investimento da indústria farmacêutica e poucos programas de combate aos vetores de transmissão dessas enfermidades ${ }^{4}$. Nas 
últimas duas décadas tem recebido uma atenção especial dos programas da OMS, com medidas de incentivo ao controle da doença nas áreas endêmicas e maior fomento na pesquisa de novos fármacos ${ }^{9}$. Nas áreas endêmicas existem inúmeros problemas, tais comoo baixo investimento pelos governantes na prevenção ea dificuldade de combate à enfermidade nas localidades isoladas ${ }^{10}$. A Guiana Francesa, área endêmica de malária ${ }^{4}$, recebeu uma série de investimentos para 0 combate à doença nos últimos anos, o que resultou na redução das taxas de contaminação pelo P.vivax em $80 \%$ e do P. falciparum em $20 \%$ de 2005 a $2016^{4,10}$. Em contraste, no mesmo período ocorreu o aumento da doença nas áreas de garimpo ilegal na floresta Amazônica ${ }^{10}$. Nos garimpos, tais como o frequentado pelo paciente em questão, imigrantes ilegais trabalham sem nenhuma assistência à saúde e cuidados básicos ou segurança para a atividade extrativista ${ }^{10}$. O paciente relatado possui residência fixa em Guarulhos, estado de São Paulo, área não endêmica de malária ${ }^{1}$, mas viajou para a Guiana Francesa e lá trabalhou por três meses. Após a evolução de febre típica, naturalmente aventouse a hipótese de contaminação pelo Plasmodium.

A malária em pacientes transplantados renais é muito pouco descrita na literatura, que relata existirem diferentes formas de contaminação para essa população especifíca ${ }^{11}$.

Assim, o transplantado pode se contaminar através da picada do inseto infectado (ciclo natural da doença) e pode evoluir mais gravemente pela maior replicação do parasita devido à terapia de imunossupressão a que está sujeito (terapia contra a rejeição do órgão transplantado) $)^{7,8}$. A doença pode, também, ser contraída pelo enxerto, quando este é extraído de doador infectado. $\mathrm{O}$ receptor do órgão infectado após o início da terapia imunossupressora apresenta disseminação do parasita, iniciando o quadro de malária ${ }^{12}$. A forma de transmissão de malária pela transfusão sanguínea não é comumno Brasil, mas deve ser citada pela prevalência em outros países.

Neste caso específico, foi afastada a possibilidade de contaminação via enxerto devido ao longo tempo decorrido desde o transplante (10 anos) e ao fato de o doador do órgão nunca ter apresentado a doença. Em todos os pacientes com relato de transmissão via órgão transplantado, os sinais e sintomas da doença ocorreram em até um ano pós-transplante ${ }^{11,13}$. Adicionalmente, sabe-se que o período crítico para infecções oportunistas ocorre nos três primeiros meses após o inicio da terapia imunossupressora ${ }^{14}$.

Em 2010, o tratamento para malária foi uniformizado pela $\mathrm{OMS}^{4}$. Esta preconizou como terapia de primeira escolha para infecção por $P$. falciparum, a terapia combinada baseada na administração deartemisinina (ACT). São exemplos de ACT atualmente empregadas a artesunato- mefloquina, a artemeter-lumefantrina, aartesunatoamodiaquina, a artesunato-sulfadoxina-pirimetamina e a diidroartemisinina-piperaquina ${ }^{15}$. A terapêutica para a malária causada por $P$. vivax é realizada com a cloroquina e seus derivados, conforme orientação da OMS. No Brasil, o Ministério da Saúde (MS) mantém a indicação da cloroquina pelo baixo perfil de resistência a droga apresentada pelo parasita ${ }^{4,6,15}$.

Segundo estudos realizados no Camboja e na Tailândia entre os anos de 2001 e 2003, surgiram cepas de $P$. falciparum menos sensíveis aos derivados do artesunato. Estes trabalhos sugerem que a administração descuidada dos fármacos (horários incorretos e dosagens incompletas) podem ser as possíveis causas da perda de sensibilidade ${ }^{16}$. Em um outro trabalho ${ }^{10}$, os garimpos ilegais de ouro foram investigados com relação a ineficácia dos derivados do artesunato (perda de sensibilidade) frente ao Plasmodium. Este trabalho verificou nos garimpos ilegais as mesmas práticas ocorridas no Camboja e na Tailândia einferiu que podem ocorrer osmesmos problemas ${ }^{10}$.

O mecanismo de ação do artesunato ainda não foi completamente esclarecido, assim como não o foi o mecanismo de perda de sensibilidade pelo P. falciparum $^{15,16}$. O paciente em questão procurou assistência médica na Guiana, como relatado anteriormente, e foi tratado com Artefan ${ }^{\circledR}$ por quatro dias. Segundo Vilaça ${ }^{16}$, o tratamento deve ser realizado por sete dias para pessoas sem comprometimentos imunulógicos ${ }^{16}$.

O paciente apresentava infecção por $P$. vivax e, conforme orientação do Ministério da Saúde, a droga de escolha seria a cloroquina ${ }^{4,7,15,16}$. O paciente utilizou a medicação inicial $\left(\operatorname{Artefan}^{\circledR}\right)$ por tempo inferior ao preconizado nas diretrizes do MS, podendo esta ser a causa da manutenção da replicação do protozoário, mesmo após a utilização do medicamento ${ }^{10,15,16}$. Há que se ressaltar, ainda, que como o paciente fazia uso de terapia imunossupressora, existe a necessidade de se prolongar o tempo de tratamento ${ }^{4,6}$.

Conclui-se, portanto, que o tratamento realizado com cloroquina é eficaz para infecções causadas pelo $P$. vivax, havendo resolução dos sinais e sintomas em três dias. No caso de pacientes transplantados renais que utilizam medicamentos imunossupressores, é de extrema importância ampliar o tempo de administração da medicação. Na abordagem sugerida por este trabalho, a primaquina usada por dezoito dias após a cloroquina mostrou-se uma excelente escolha.

\section{Referências}

1. Brasil. Ministério da Saúde. Malária [Internet]. Brasília 1953 - 2017. Disponível em:http://portalms.saude.gov.br/saude-de-a-z/malaria/descricaoda-doenca.

2. Camargo EP. Malária, maleita, paludismo. Cienc. Culto. [Internet]. 
2003 Jan [citado 201726 de agosto]; 55 (1): 26-29. Disponível em: http://cienciaecultura.bvs.br/scielo.php?script=sci_arttext\&pid=S0009$67252003000100021 \& \operatorname{lng}=$ pt.

3. Costa AP, Bressan CS, Pedro RS, Valls-de-Souza R, Silva S, Souza PR et al.Diagnosticando Malária em Área Endêmica de Dengue na ExtraAmazônia, Revista da Sociedade Brasileira de Medicina Tropical, set-out, 2010:43(5):571-574.

4. World Health Organization.World Malaria Report 2016: Summary. (WHO/ HTM/GMP/2017.4). Geneva, 2017.

5. Lapouble OMM, Santelli ACFS, Muniz-Junqueira MI. Situação Epidemiológica da Malária na Região Amazônica Brasileira, 2003 a 2012. Rev Panam Salud Publica. 2015: 38(4):300-6.

6. Brasil. Ministério da Saúde. Secretaria de Vigilância em Saúde. Departamento de Vigilância Epidemiológica. Guia prático de tratamento da malária no Brasil/Ministério da Saúde, Secretaria de Vigilância em Saúde, Departamento de Vigilância Epidemiológica. - Brasília: Ministério da Saúde, 2010.

7. Costa, S.D. Incidência de Doenças Tropicais e Lesão Renal Aguda em Pacientes Submetidos a Transplante Renal[dissertação]. Universidade Federal do Ceará - Faculdade de Medicina 2015.

8. Fischer L, Sterneck M, Claus M, Costard-Ja"ckle A, Fleischer B, Herbst H, Rogiers X, Broelsch CE. Transmission of Malaria Tertiana by MultiorganDonation, Clin Transplantation 1999: 13: 492-496.

9. Reis,ACS de M,Borges DPL, CruveloD’ÁvilaVG de F, Barbosa MS, Ternes YMF, Santiago SB, Santos RS.,et al. O Cenário de Políticas Públicas do Brasil Diante do Quadro das Doenças Negligenciadas, SAÚDE \& CIÊNCIA EM AÇÃO, Revista Acadêmica do Instituto de Ciências da Saúde, Agosto-Dezembro 2016: 3 (1).

10.Douine M, Musset L, Corlin F, Pelleau S, Lazrek Y, Mutricy L, et al.Paludisme et OrpaillageIllegal en Guyane: Un EnjeuMajeur de Sante Publique. Bulletin EpidemiologiqueHebdomadaire - BEH, Saint-Maurice (Val de Marne): Institut de Veille San- Itaire, 2017:6, 102-114.

11. Sousa, SR, Galante NZ, Barbosa DA, Medina Pestana JO. Incidência e Fatores de Risco Para Complicações Infecciosas no Primeiro Ano Após o Transplante Renal, Jornal Brasileiro Nefrologia.2010: 32(1):77-84.

12. Simforoosh N, Shemshaki H, Nadjafi-Semnani M, Sotoudeh M. Living Related and Living Unrelated Kidney Transplantations:A Systematic Review and Meta-Analysis, World Journal Transplant 2017 April 24; 7(2): 152-160

13. KuteVB, Vanikar AV, Shah PR, Shrimali JD, Gumber MR, Patel HV, Modi PR, Trivedi HL Postrenal Transplant Plasmodium Vivax Malaria: Neglected and Not Benign, Parasitol Res.2013: 112:1791-1793.

14.NassenS. Malaria With Leukopenia in a Renal Transplant Patient, Transplantation, 2011: 91 (11).

15. LeiteFHA. Malaria: From Old Drugs to New Molecular Targets, BBR - BIOCHEMISTRY AND BIOTECHNOLOGY REPORTS, Jul./Dez2013: 2 (4): 59-76.

16. VilaçaMJL, Artemisininas no Tratamento da Malária: Perspectivas Futuras [Dissertação]. Faculdade de Medicina - Universidade do Porto 2010.

17.Rey L. Bases da Parasitologia Médica. $3^{\text {a }}$ Ed. Rio de Janeiro: Editora Guanabara Koogan.;2010. 